\title{
Subjectivité poétique, dialogisme et transitivité
}

\author{
CARMEN POPESCU
}

\begin{abstract}
Poetic subjectivity, dialogism and transitivity. The paper studies the interplay between three key-concepts, subjectivity, dialogism and transitivity, with reference to the recent transformations of the poetic discourse, especially in the context of Romanian postmodernism. Poetry is traditionally considered the most subjective of genres, with subjectivity being perceived as a psychological element, with some philosophical overtones (particularly within Romanticism). Modern theories of enunciation and discourse can shed new light on this presupposition, by revealing the linguistic dimension of the subjective feature. By the same token, subjectivity is revealed as being intimately intertwined with dialogism. Although implicit in any type of discourse (as in Bakhtin's account), dialogism can also be deliberately emphasized. The paper is concerned with the more explicit dialogization of contemporary poetic discourse. Transitivity pertains to the communicative dimension of poetry. Borrowed by Gheorghe Crăciun from Tudor Vianu, who distinguished between the reflexive and the transitive function of language, transitivity is applied by Crăciun to modern and postmodern poetry. In the corpus of Romanian poetry analysed in this article, the self is being deconstructed and reconstructed on new coordinates and interpersonal and intertextual dialogue is being employed as a means of resistance to ideology and social engineering. In the poem "Without them", Mariana Marin writes a poetic homage to the German poets in Romania, who have influenced her towards a "committed subjectivity" and away from a neo-Romantic, narcissistic subjectivity. Letiția Ilea grafts reported discourse on her pseudo-confessional poem "A beautiful spring day. In the fields" in order to bring attention to the failure of genuine dialogue and the inner, polyphonic theatricality of the self. Dumitru Crudu in the poem "dimitrie" designs confessional personae for himself and solicits the readers' empathy, while Ioan Flora, in "Poetry is a document, I said to myself", resorts to metapoetry, in search of a new poetics, of a more "truthful" and "just" type.
\end{abstract}

Keywords: subjectivity, dialogism, transitivity, Romanian postmodern poetry

\section{Introduction}

Le discours poétique contemporain se remarque par une grande richesse de modalités pour configurer l'identité du locuteur et pour communiquer la subjectivité poétique. La dernière représente, traditionnellement (ou, plus 
précisément, à partir du romantisme), la substance et la texture essentielle du lyrisme : «lyrique », «c'est-à-dire [...] une poésie d'expression personnelle vouée à l'épanchement de la sensibilité. Nous identifions donc la poésie à une énonciation en première personne et à un contenu affectif » (Jenny $2003: 1$ ).

Si la théorie traditionnelle des genres appréhendait la subjectivité premièrement en tant qu'auto-expression du « moi », le développement récent de la pragmatique, de l'analyse du discours et des théories de l'énonciation offrent une nouvelle base technique pour l'investigation des subjectivèmes poétiques. Benveniste affirmait que « Le langage est la possibilité de la subjectivité [...], et le discours provoque l'émergence de la subjectivité » (1966 : 263). A part l'(inter) subjectivité intrinsèque et constitutive au langage, analysée en détail par les linguistiques de l'énonciation, on doit tenir compte aussi de la subjectivité actualisée et marquée dans le discours. Ces subjectivèmes sont plus ou moins présents en fonction du type de texte ou de discours : «le taux de subjectivité varie d'un énoncé à l'autre » (Kerbrat-Orecchioni 2002 : 82). Selon le « degré de présence / effacement du sujet parlant », les textes produisent des « effets de subjectivité $\gg$ (Sarfati 2009 : 46-47) différents.

Tout comme la subjectivité, le dialogisme peut être envisagé sur deux plans : comme inhérent et constitutif au discours ${ }^{1}$, et comme ostentatoire et délibéré, présent dans la structure de surface du texte, pour des raisons expressives et communicatives diverses. La poésie contemporaine explore par ses propres moyens la crise du sujet, mais aussi la problématique de l'intersubjectivité et les difficultés de la communication interpersonnelle dans le contexte de l'aliénation (post)moderne. L'antonyme de la subjectivité n'est pas l'objectivité, mais plutôt le dialogue. Le dialogisme poétique devrait être abordé dans la perspective de l'intertextualité mais sans en ignorant l'intersubjectivité que les Tel-Quelistes avaient niée (cf. Kristeva 1969 : 85). L'hypothèse poststructuraliste de la « mort de l'auteur » (cf. Barthes 1984 [1968]) n'est plus suffisante pour comprendre l'engagement communicatif des poètes contemporains (cf. Popescu 2014).

Pour ce qui est du troisième concept, la « poésie transitive » est vue comme «l'iceberg de la poésie moderne » (Crăciun 2002). C'est une théorie qui puise sur la distinction de Tudor Vianu entre une fonction réflexive et une fonction transitive du langage. D’après Vianu, « celui qui parle communique et se communique. Il le fait pour les autres et pour lui-même » (1988: 13). Le paradigme traditionnel du lyrisme semble mettre un signe d'égalité entre la fonction poétique et la fonction réflexive du langage, tandis que dans la poésie

1 « L'expression d'un énonce est toujours, à un degré plus ou moins grand, une réponse [...] $\gg$ (Bakhtine $1984: 299)$. 
POPESCU

contemporaine, qui ne craint pas la comparaison avec la prose, la fonction transitive (ou conative, dans le modèle jakobsonien de la communication) devient dominante. Le comparatiste Jean Bessière a montré que par la « figuration de la transitivité sociale », « l'œuvre possède une propriété critique $\gg(2008: 78)$.

\section{Déconstruction et reconstruction du moi dans le postmodernisme roumain : résistance et engagement}

Le statut du moi dans la poésie postmoderne est très complexe. En Roumanie, les poètes des années 80 voyaient dans les procédés du postmodernisme un moyen de résister à la pression idéologique extrême générée par le contexte totalitaire. Ils ont donc investis ces procédés d'une force subversive supplémentaire. L'attitude envers l'individualisme ne pouvait être qu'ambivalente : d'une part, les poètes tentaient de résister aux injonctions dé-personnalisantes de la massification communiste, d'autre part, ils étaient conscients des contradictions inhérentes à l'individualisme moderne. La tradition chrétienne orthodoxe, qui met l'accent sur la personne et la communion des personnes ${ }^{2}$, a eu elle-même un impact important sur la poétique configurée chez certains des poètes analysés.

Les jeunes poètes croyaient en une soi-disante résistance culturelle qui ne pouvaient être que limitée, par rapport aux rigueurs politiques du moment, mais qui était, pour les écrivains, la seule modalité accessible pour préserver la dimension esthétique du discours tout en évitant le danger de la censure totale. Par conséquent, la force illocutoire du discours poétique devrait être considérablement accrue, quoique dans les cadres de l'expression ésopique, allusive et indirecte. Le « nouvel anthropocentrisme » postulé par Alexandru Mușina (1995 : 165) était favorable à un type de poésie dont le coefficient de transitivité était très haut. Une nouvelle poétique prend contour, où «l'intensité stylistique » a été substituée parl' « intensité de la communication » (Mușina, apud Crăciun 1999 : 170). L’ouverture vers l'altérité accompagne la quête de l'authenticité. Dans une interview (Gogea 1999 : 62), Dumitru Crudu établit une connexion entre le sentiment de la fausseté de sa vie et sa solitude. La poésie de qualité, croit-il, a toujours été créée à cause d'une forme quelconque de non-solitude expérimentée par les créateurs.

2 Dans les mots de Dumitru Stăniloaie (1987), il s’agit du dialogisme constitutif de la condition humaine en tant que visage de la divinité. 
Ioan Bogdan Lefter a parlé d'un « retour du moi de l'auteur » dans l'écriture postmoderniste, qui s'exprime par la « re-biographisation des personnes grammaticales par un nouveau engagement existentiel » (Lefter 1995 : 170). La présence plus évidente du moi dans la poésie (particulièrement dans sa dimension extérieure, sociale, autobiographique, réaliste) remplit un fonction polémique et métapoetique. L'identité du poète postmoderne est devenue un épiphénomène, un puzzle, une mosaïque de fragments, d'échos et d'influences, des voix étrangères parfois. Pourtant, par les stratégies de dialogisation et de transitivité, ces poètes urbains désabusés font un effort de résister aux effets de réification de la civilisation contemporaine. Pendant la dictature communiste, l'hybridation stylistique avait le rôle de contester la fausse uniformité imposée par l'idéologie et la propagande. Apres la chute du communisme, le mélange des voix et de registres est à la fois un reflet de la réalité extratextuelle hétérogène et une stratégie d'opposition aux mécanismes commerciales de la société de consommation.

Un sens plus fort de la transitivité sociale en tant que vocation poétique devenait visible, surtout chez les représentants de la minorité allemande de Roumanie, Aktionsgruppe Banat (Le groupe d'action de Banat), qui professaient « une subjectivité engagée » (Fromm 1979: 3) ${ }^{3}$. La subjectivité n'est donc pas refusée ou exilée hors de l'univers poétique, mais assumée et intégrée dans un réseau social plus vaste, incluant la solidarité. Tandis que les poètes roumains étaient plus préoccupés de prendre distance par rapport au réalisme socialiste et les autres produits du discours officiel, leurs congénères allemands n'hésitaient pas d'approprier les clichés de la propagande - dont l'engagement des écrivains était un des mots-clé - pour leur donner de nouvelles connotations. Mariana Marin (1956-2003), qui a été persécutée par la police secrète et même censurée, a évoqué le groupe et leur influence bénéfique dans le poème $\ll$ Fără ei $\gg(\ll$ Sans eux $\gg)$ :

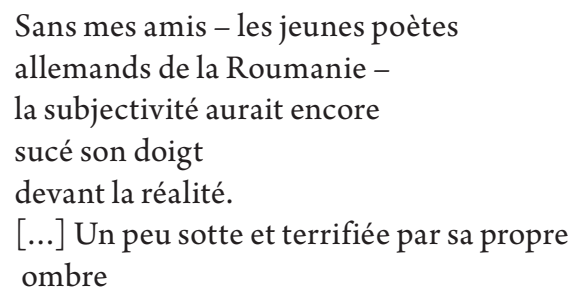

3 Un autre détail significatif est leur option explicite pour le dialogue. Les séances tenues par les jeunes poètes avaient le titre «Au commencement c'était le dialogue » (Am Anfang war das Gespräch) (cf. Bernic 2012). 
POPESCU

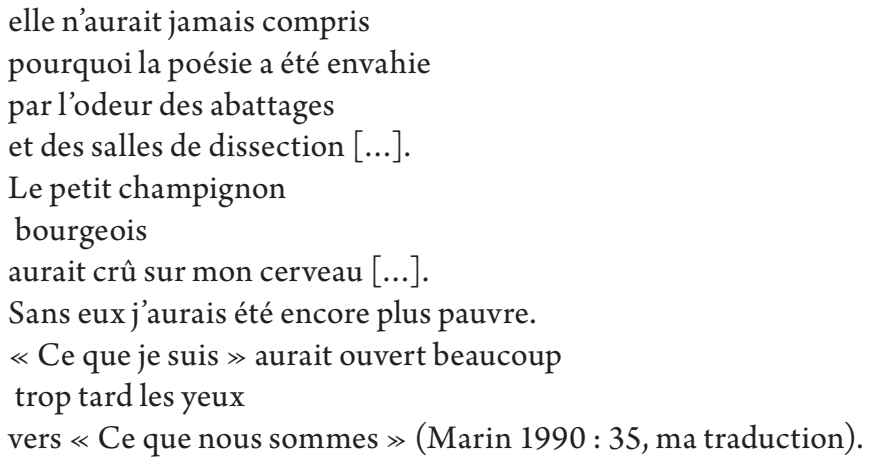

Le dialogisme intertextuel mais aussi interpersonnel qui la liait à ses amis du groupe allemand a donc joué un rôle considérable pour rendre sa relation avec le public plus transitive, ça veut dire plus engageante et engagée, plus transparente et directe. Leur influence l'a aidée à dépasser un modèle néoromantique ou haut-moderniste de poésie. Dans cette confession-hommage, Marin se montre consciente de la nature ontologique du dialogisme, par le jeu des pronoms et des formes du verbe être. Les périphrases «Ce que je suis » et « Ce que nous sommes » sont des personnifications (tout comme «la subjectivité »), et sont certainement figurées comme des aspects divergents de la personnalité créatrice. Marin rompt avec la poétique juvénile et egocentrique, en optant pour une poétique dialogique-transitive, du moi-en-relation, médiée par l'intertexte.

\section{Théâtre intérieur et polyphonie (voix des autres)}

Les stratégies de dialogisation dans la poésie contemporaine roumaine sont diverses : polyphonie ou multiplication des voix, des points de vue et des «masques » stylistiques, complexité de l'appareil énonciatif - deixis personnel, alternance de l'embrayage avec le non-embrayage (les énoncés « sans locuteur »). Les poètes thématisent explicitement l'identité poétique et manifestent un intérêt majeur pour tous les aspects de la communication littéraire. Chez beaucoup de ces auteurs, le poème devient souvent l'espace d'une performance - une externalisation du «théâtre » intérieur. Les éléments de nouveauté résident dans la prise en charge des points de vue (ce qui entraine la responsabilisation du locuteur), et à la fois dans la co-construction dialogique de l'identité, par l'intertexte et par la complicité du lecteur. 
Subjectivité poétique, dialogisme et transitivité

Un poème par Letiţia Ilea (née en 1967), intitulé « une belle journée de printemps. en plein champ » est un exemple assez typique pour cette modalité d'exhiber le monde intérieur du sujet parlant :

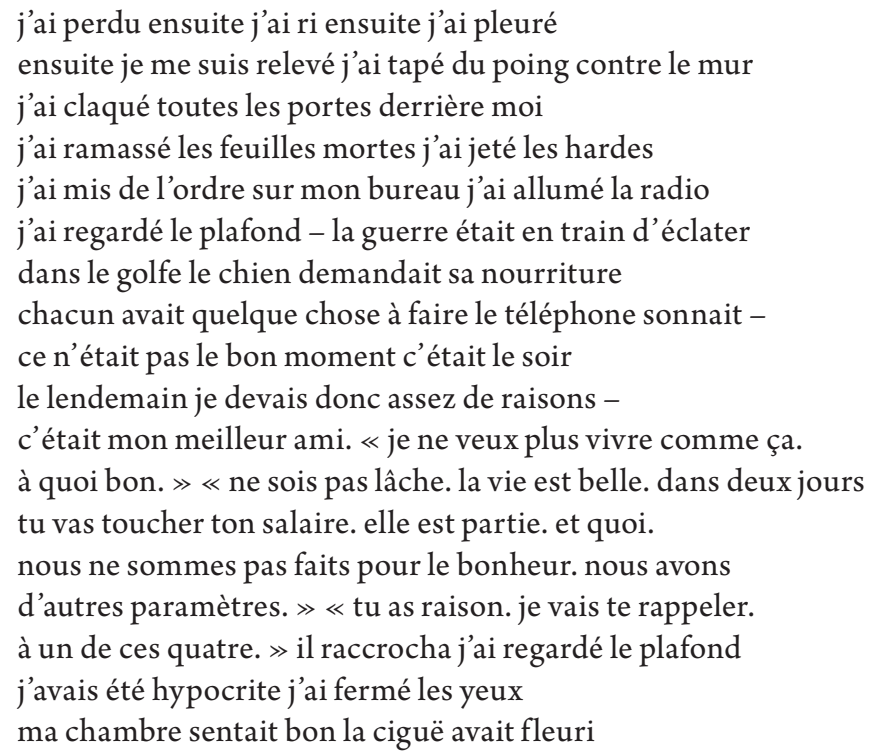

(Traduit du roumain par Letiția Ilea, dans Bodiu, Bucur, Moarcăș 1999 : 307. )

Letiția Ilea est une représentante de la nouvelle vague poétique des années 90 (une branche qui est souvent étiquetée de néo-expressionniste). Comme ellemême l'avoue, écrire de la poésie a pour elle une fonction d'exploration et de découverte de l'espace intérieur : «j'écris pour me parcourir, pour m'explorer, pour faire la paix avec moi-même $\gg^{4}$. Mais ce domaine de l'intimité subjective s'avère structuré dialogiquement, étant donnée la profusion de citations, de répliques, d'échos et autres formes d'intertextualité qui attirent le regard.

Le locus amoenus configuré par le paratexte et le dernier vers institue un idyllisme / bucolisme minimaliste, rendu de façon ironique (modalisation qui crée une distance entre l'énoncé et l'énonciation, le dit et le dire), suggérant le besoin d'évasion. Le référent absent mais implicite serait l'angoisse ou la déception, à reconstituer à travers l'atmosphère, l'usage de la répétition lexicale (indice d'obsession), le parallélisme syntaxique, et l'enchainement d'actes de

4 http://www.roumanie.com/La-poetesse-Letitia-ILEA-A00705.html. 
POPESCU

langage constatifs où tous les verbes sont à l'indicatif, passé composé (décrivant une série de gestes et de réactions émotionnelles) : «j’ai perdu $\gg[$ le verbe est transitif mais l'objet direct manque], «j'ai ri », «j'ai pleuré $\gg$, etc. Le déictique de première personne établit, naturellement, une déixis égocentrée. Les déictiques de temps suggèrent la rétrospection mais la situation d'énonciation, l'ancrage sont quand même vaguement déterminés. Le soliloque poétique a l'apparence d'un flux de la conscience, se transformant peu à peu en dialogue intérieur - avec soi-même et avec les voix des autres, intériorisées. La greffe discursive survient par le discours rapporté, ici dans la forme des fragments guillemetés, une source importante de polyphonie textuelle (cf. Flottum 2002). L'équivoque ou l'ambiguïté sont probablement des effets prémédités : y-a-t-il une sortie, y-a-t-il de l'espoir (« la ciguë avait fleuri $»)$, en dépit de l'imaginaire dysphorique dominant, ou au contraire, l'évasion se prouve une fausse évasion (《j'avais été hypocrite j’ai fermé les yeux »), possible seulement au niveau de l'imagination?

Il s'agit, apparemment, de quelqu'un qui a grand besoin de consolation et de tendresse, et qui est obligée, à un certain point, de consoler elle-même un ami. Mais la situation d'interaction est à peine esquissée. Le traitement textuel du thème ouvre la possibilité de l'empathie mais semble la bloquer, à la fois, à cause, peut-être, du solipsisme duquel l'auteur elle-même est consciente. L'échec de la communication interpersonnelle pourrait être suggéré par les clichés qui sont employés pour conseiller ou encourager l'ami. C'est la sphère de la doxa et des injonctions impersonnelles mais autoritaires agissant au niveau de l'inconscient. Le réservoir commun des stéréotypes transforme la personne dans une machine à communiquer, qui ne pourrait désormais ni prétendre d'être écoutée et comprise, ni de connaitre et d'accepter intimement l'autre. Néanmoins, si la communication représentée est un échec (un faux dialogue), la communication enchâssante, entre le locuteur et le lecteur est préservée, est l'effet de transitivité est même augmenté.

\section{Les masques de la confession}

Une influence importante sur la poésie roumaine contemporaine a été la poésie confessionnelle (confessional poetry) américaine (Robert Lowell, John Berryman, Sylvia Plath, Anne Sexton, etc.). La subjectivité, les problèmes d'identité, les profondeurs abyssales du moi et bien sûr, les traumas psychiques (l'aliénation, la perte de soi, le malaise et le mal de vivre) occupe une partie majeure dans ce type de poésie. 
Mais il faut remarquer les modalités par lesquelles ce modèle-là est subverti et transformé. Dans la variante roumaine de la poésie personnelle (cf. Maio 2005), l'inscription de la subjectivité (ça veut dire, le narcissisme poétique) est contrebalancée par les stratégies dialogisantes et transitivisantes qui articulent une nouvelle conception de la communication poétique. L'altérité (la voix de l'autrui) est greffée sur le discours propre par le discours rapporté et représenté, par la citation littéraire, les échos et les allusions (donc l'intertextualité) et par l'interpellation du lecteur d'une manière colloquiale et amicale. Ces procédés conversationnels sont générateurs de polyphonie ou multivocalité, parce que la voix et la réponse de l'allocuteur sont introjectées et anticipées dans le texte. L’ambiguïté propre au discours poétique fait difficile l'attribution des voix aux quelconques énonciateurs, mais c'est précisément cette complexité et diversité que le poète contemporain veut exploiter, afin de mieux impliquer le lecteur dans le jeu communicatif. Je veux marrêter maintenant sur un poème par Dumitru Crudu (n. 1968), intitulé « dimitrie », qui fait partie du volume Falsul Dimitrie (Le faux Dimitrie, 1994). Les stratégies d'ethos (ou image rhétorique du locuteur) employées par Ilea sont en contraste avec la mythologisation paradoxale du moi chez Crudu, réalisée par le recours persistent au nom réel de l'auteur ${ }^{5}$. Cette stratégie peut être lue comme une allusion parodique au biographisme dogmatique de la génération postmoderne roumaine, qui pourtant a des conséquences intéressantes dans le domaine de la communication poétique :
je suis le rat je suis le ver
je suis le papillon qui vole dans l'air
nous tous nous sommes ceux qui marchons
sur la terre et qui rampons sur les coudes nos
voix ne sont pas audibles sont faibles faibles
mais parfois nous nous voyons je suis le rat
qui sort sa tête d'un vieux trou. je sais
que je suis moche et à cause de ça je souffre énormément

Même concernant cet aspect il y a un certain écart, parce que «Dimitrie » est une variation onomastique de «Dumitru ». En fait, la version choisie pour le volume de 1994 rappelle le non du Saint Dimitrie (Demetrios) de Thessaloniki, un très important saint-martyre du christianisme orthodoxe, donc l'archétype de «Dumitru ». C'est pour ça, peut-être, que le poète se décrit comme un «faux » Dimitrie : parce qu'il est si loin du modèle idéal, de l'archétype. Le saint est celui qui a surmonté la « fausseté » de l'existence païenne, idolâtre, pour l'identité du converti chrétien, prêt à se sacrifier pour l'amour de Dieu et de ses semblables. Le «faux Dimitrie » est celui qui déplore son incapacité de se sauver d'une existence inauthentique, minée par l'individualisme. 
POPESCU
et moi je suis le ver
qui grouille dans la bouse
et moi j'ai des complexes mais le soir vient et il me prend
dans ses paumes et il joue avec moi nous tous nous sommes
solitaires solitaires et nous souffrons énormément
à cause de ça et moi je suis le papillon qui
vole là-haut et moi je suis seul seul et j'ai
peur parfois quand je me regarde dans la glace
mais le soir vient et l'été vient et nous
sortons dans les champs et nous écoutons vos
chants et ils nous plaisent énormément
je suis le rat je suis le ver
je suis le papillon qui vole dans l'air
et on aime à la folie
vos chants absurdes

(Dumitru Crudu, « dimitrie », traduit par Radu Stoenescu dans Bodiu, Bucur, Moarcăș 1999: 320)

L'introspection, même dans un style ironique ou désinvolte, se communique par les métaphores de l'identité - « je suis le rat, je suis le ver » - et détermine des effets paradoxales. A côté de l'emphase théâtrale, l'humilité du psalmiste est aussi présente ${ }^{6}$. L'exploration du soi suscite l'horreur du sujet devant sa propre laideur. Malgré une certaine impression d'impudeur extrême que peut faire cette confession, les détails autobiographiques qui devraient (dans la logique de la poésie confessionnelle et personnelle) soutenir l'autoportrait négatif manquent. J'y vois une subversion ironique de l'impératif autobiographique promu par ses collègues de génération. La poésie personnelle préférée par Crudu s'universalise par des moyens quasi-impersonnels (soutenant un « moi » générique), mais sans retourner, pour ça, à une poétique moderniste. Il y a de la confession, ou mieux dit, de l'auto-analyse, qui est impitoyable, par sa dureté, mais adoucie, quand même, par la façon dont le poète fait de sa situation un paradigme de la condition humaine : « nous tous nous sommes ceux qui... ». Une autre modalité par laquelle l'auto-dissection est, dans une certaine mesure, adoucie, est par des vers comme $<$ je suis le papillon qui vole dans l'air», qui assurent un contrepoids symbolique positif à l'hypostase identitaire dominante dans le texte, qui est métaphorisée dans un registre de la négativité (《rat », « ver »).

6 Cf. le Psaume 21, « Et moi, je suis un ver, pas un homme, raillé par les gens, rejeté par le peuple », http://www. ndweb. org/ psaume/21/index.html. 
Le texte dimitrie abonde en marqueurs affectifs et axiologiques de la subjectivité. C'est un texte transitif, orienté vers l'altérité (et traversé par celle-ci), mais à la fois egocentrique et narcissiste, même histrionique ; un poème plutôt monologique que vraiment dialogique (au sens bakhtinien). Des aspects intéressants sont : l'embrayage très marqué (en particulier la deixis de personne), et les deux contenus du pronom personnel nous, qui sont évidemment contradictoires. Le premier nous (tous) a une fonction argumentative-persuasive, et suggère une généralisation peut-être abusive de la condition du sujet (la solitude), ou une instrumentalisation d'un cliché (tout homme est seul, chacun meurt seul, tout le monde a peur, etc.). L'attitude impliquée (jouée, mise en scène) est un désir presque désespéré d'être accepté et compris par tout le monde. Le deuxième « nous » construit une solidarité beaucoup plus restreinte mais qui reste, en fin de compte, non-précisée. L'opposition est cette fois entre un «nous » mystérieux (un groupe quelconque, une paradoxale communauté de solitaires avec qui «dimitrie » s'identifie) et le reste, désigné par un vous auquel on associe des « chants » qui d'une part « plaisent énormément » au premier groupe et qui d'autre part sont « absurdes », ce qui implique que le plaisir devrait être lu de manière antiphrastique, ironique. $\mathrm{Ce} \ll$ nous $\gg$ inclusif et abusif ${ }^{7}$ semble se transformer, finalement, dans un pluriel de la (fausse) modestie, un autre masque pour le moi narcissique du poète, dont l'obsession de soi est en fait la vraie maladie et la laideur qu'il déplore lui-même. Les personae poétiques qu'il crée ne sont pas des stratégies élusives, destinées à cacher la « vraie » identité (qu'il ne connait pas lui-même) : elles sont plutôt des moyens d'exploration de soi (et de l'autrui).

\section{Subjectivité créatrice négociée et soumise au débat public}

La scène énonciative du poème contemporain absorbe tous les thèmes possibles et leur confèrent une dimension dialogique. L'univers préféré des postmodernes, celui du méta-discours / méta-énonciation (l'art poétique dans tous ses états) ne fait pas exception, étant d'une part personnalisé par la saillance, l'hyperprésence ou l'emphase du locuteur ${ }^{8}$ (une emphase paradoxale, menant en fin de compte à une diminution du soi, puisque ce sont surtout ses impuissances,

7 Qui est substitué, d'ailleurs, dans le dernier vers de la traduction française, par le « on » impersonnel : «Et on aime à la folie...».

8 Souvent identifié, en vertu de l'authenticisme et du biographisme de cette génération, au sujet parlant, à la personne empirique de l'écrivain. 
POPESCU

incertitudes et inquiétudes qui sont dévoilés) et d'autre part étant ouvert au débat, entrainant des réponses et des contributions de la part du récepteur. Par exemple Ioan Flora (1950-2005) décrit minutieusement, dans le poème « Poezia-i document, mi-am zis » (« La poésie est document, me dis-je »), les processus intérieurs accompagnant la recherche d'une nouvelle poétique : monologue rapporté, délibération intime, lutte entre les voix qui assiègent la conscience et le sous-conscient ; mais il semble à la fois étrangement détaché de tout ce travail méta-poétique, comme s'il voulait savoir si la poésie pouvait vraiment être document; et le lecteur est conçu comme un facteur actif dans le dialogue implicite. Pour Flora, la notion réaliste de la poésie-document fonctionne comme une « dislocation programmatique du logocentrisme moderne $\gg($ Parpală $2011: 76)$ :

\section{$[\ldots]$}

Tu avais débattu des après-midi entiers, sur une possible

et juste poétique :

le vendeur, le mécanicien, le comptable, le proxénète, la tisseuse, l'entraineuse, la caissière, le psychiatre, la femme de ménage,

la femme aux lys cirés appliqués sur le chemisier, l'addition, l'horaire des trains, les mauvais vins, l'aspect de foire de la métropole, les parfums et l'odeur et l'aigreur de la sueur,

\section{$[\ldots]$}

la poésie est document, tu exagères peut-être

si je lis encore un vers, j'en crève

[...] je mangerais

de la viande crue, hachée, poivrée, celle-là

qui se trouve sur la table.

Pourquoi tu trembles, ma main tremble, je hurle, [...] essaie de redresser ton épine dorsale, tu es un être social, tu vis, tu vis encore avec les autres. 
Subjectivité poétique, dialogisme et transitivité

Lis les journaux, donne de coups de fil, parcours les rues, appuie fort sur la porte, achète-toi un peigne, écris sur les lieux :

ce sont tes droits, ce sont tes uniques

obligations.

Ton bon nom chatouille et défie

sur le boulevard Magheru,

Parmi chapeaux et chemisiers,

$[\ldots]$

tu es un être social,

tu manges avec les autres, tu te couches

à côté des autres ;

tu restes du côté des choses et des lieux

concrets,

me dis-je.

(Ioan Flora, « La poésie est document, me dis-je », La métaphore trahie, 2004 : 23-27, traduit par Paul Miclău)

Le locuteur apparait disposé à affaiblir volontiers sa propre position afin de rendre plus fort l'allocutaire qui pourrait d'ailleurs l'aider dans sa quête pour une poétique nouvelle mais surtout vraie, adéquate pour les besoins des partenaires du dialogue. Ce type d'argument méta-énonciatif et méta-dialogique (qui est implicite) détient la place principale parmi les autres arguments, portant sur la relation du poème au monde environnant. L'énonciateur représenté est en train de négocier, mais d'une façon subtile, cette poétique de la poésiedocument. Ce n'est pas une affectation, ou une nouvelle rhétorique dérivant du geste démonstratif de - disons - tordre le cou à l'ancienne rhétorique. La force expressive de cette tonalité réside précisément dans l'effet de sincérité qu'elle produit, tandis que la force illocutoire dérive de l'inscription de la transitivité dans une formule d'auto-dialogisme : le dialogue avec un énonciateur non-précisé, peut-être la conscience (mais une conscience chorale, une voix collective), qui lui rappelle son statut ontologique d'être social. En même temps, l'ironie résultant des difficultés majeures inhérentes à la posture énonciative de poète engagé ne pourraient pas échapper au lecteur, surtout celui qui place le texte dans le contexte de l'entier corpus poétique de Flora. Le poème fait partie du volume Lumea fizică (Le monde physique, 1977), apparu dans un « contexte de surenchère métaphorique », la poésie roumaine étant, à cette époque-là, « sous la tutelle de la génération de Nichita Stănescu, Cezar 
Baltag, Ana Blandiana, Ion Gheorghe » (Gheorghe Crăciun, in Flora, 2004 : 151).

La poésie en tant que document, la base de cette « possible / et juste poétique $\gg$, serait plus réaliste, plus démocratique, moins élitiste, plus inclusive avec les thèmes et les sujets à traiter mais aussi avec les personnes auxquelles elle s'adresse et lesquelles sont considérées dignes de figurer comme « personnages » dans ce type de poème (du vendeur à la femme de ménage). Par conséquent, la fonction référentielle du langage sera privilégiée, au détriment de la fonction expressive/ subjective et au détriment de la métaphore et de la connotation en général. Pourtant, le poème-manifeste contient certains indices que l'option pour une poétique dénotative, de degré zéro n'est pas tout à fait sans équivoque ou sans hésitation. Il s'agit de l'image de la viande crue, métonymie du « naturalisme » auquel l'auteur tâche de se convertir mais qui semble assez répugnante (le locuteur serait-il vraiment enthousiasmé par l'idée de manger cette sorte de nourriture ?) et du fait qu'il doit se convaincre luimême qu'il est un « être social » et qu'il reste « du côté des choses et des lieux/ concrets ». La nouvelle poétique se prétend plus « juste » (ce qui, dans les termes de cette analyse, pourrait être traduit comme plus «dialogique $\gg$ et plus «transitive ») mais elle n'est pas exempte de contradictions : le « matériel » humain se trouve sur le même plan que les objets du monde physique, et l'effet de réification des gens ne peut pas être évité. L'énumération ${ }^{9}$ a un effet d'aplanissement. Parfois, le « nouvel anthropocentrisme » clamé par Mușina se transforme, subrepticement, et malgré les meilleurs intentions des poètes, dans une subtile misanthropie. Je crois que Ioan Flora était tout à fait conscient de ces apories et qu'il en fait sujet de débat intérieur mais aussi de débat public.

Mușina (1995 : 165) parlait du «populisme des postmodernistes ». Dans un sens plus profond, il s'agit de la métacommunication (cf. Popescu 2013) : la transitivité marquée à laquelle les poètes appartenant à ce paradigme font recours imprègne leur discours d'une dominante phatique, modelée après les cérémoniaux et les rituels de l'interaction quotidienne. C'était une façon de normaliser et d'humaniser la diction poétique. En plus, cette mutation dialogique dans le lyrisme contemporain intervient comme une énergie dépoétisante et orientée vers la clarification du «message », si l'on tient compte que la cible de la polémique implicite est la difficulté (ou l'élitisme)

9 L'énumération est un procédé stylistique fréquemment employé par Flora dans ses premiers volumes. Dans la postface de la Metaphore trahie, Crăciun parle d'un « nouveau principe d'organisation poétique (principe essentiellement antipoétique), celui de la liste, réalisée dans un quasi-maniacal plaisir de l'énumération » (in Flora 2004 : 153). 
moderniste, maintenant considérée par les poètes comme un modèle révolu. Il y a, indéniablement, de la difficulté postmoderniste, mais elle n'est pas nécessairement du type sémantique et syntaxique comme celle pratiquée par les générations antérieures. C'est surtout la hiérarchisation des voix et des discours impliqués dans le dialogue implicite et explicite qui pose des problèmes pour le lecteur ou, mieux dit, qui provoque une réaction créatrice de la part de celui-ci. On trouve un exemple d'indécidable énonciatif dans les trois vers : « la poésie est document,/ tu exagères peut-être/ si je lis encore un vers, j'en crève ». (Flora $2004: 26$ ) Chacun des vers pourrait appartenir à une voix (ou un énonciateur) différente : le poète, sa conscience (critique), le lecteur. Mais les voix font partie de la polyphonie intérieure et fonctionnent comme une preuve que la subjectivité est elle-même diverse, un «éventail » de possibilités expressives ou même cognitives. Le soliloque traditionnel du poète (que l'autorité du modèle romantique l'a transformé en canon et standard de la poésie en général) subit une métamorphose, étant substitué, en fin de compte, par le dialogue implicite et par le discours poétique polyphonique.

Dans ce type de discours, le lecteur n'est plus conçu comme une fonction textuelle ou un agent qui reproduit par décodage le sens codifié par le texte, mais comme une personne vivante, un partenaire de dialogue, qui a des responsabilités et des droits communicatifs, y compris le droit de se montrer incompréhensif, ou moins réceptif. Au lieu de s'assumer le risque, du moins en théorie, de rester seul, dans sa subjectivité incommunicable, le poète préfère les compromis de l'accessibilité poétique (très relative, d'ailleurs) et par cela prolonge la conversation incessante dans laquelle il s'engage, avec la tradition et les codes littéraires (le dialogue-intertexte) et avec les lecteurs.

\section{Conclusions}

La ré-personnalisation du discours poétique a été une réaction compensatoire vis-à-vis de l'autorité du modèle (néo)moderniste qui était en quelque sorte déshumanisé et trop élitiste pour les nouvelles conditions sociales. Après l'hégémonie de l'impersonnalité (dans le sillage de Mallarmé ou T. S. Eliot), ily a donc un retour du moi dans la poésie, mais d'une manière tout à fait différente par rapport à la tradition romantique, qui focalisait une forme particulière de subjectivité.

La réinsertion du moi dans la poésie roumaine, avant et après la chute du communisme, survient par des mécanismes tout-à-fait prémédités de dialogisation et de transitivité. Dans la poésie transitive, la subjectivité est thématisée, apportée au niveau de surface du texte et même proposée comme 
POPESCU

thème de débat, dans la mesure où le poème est transformé dans un espace dialogal / dialogique, le lecteur étant invité d'être co-créateur et de manifester empathie envers ce travail de déconstruction et reconstruction du moi poétique et du moi réel / empirique. Le dialogisme et la transitivité pourraient être associés dans ce cas à la notion de responsabilité énonciative, qui est d'ailleurs une nouveauté dans le contrat poétique. (Dans la théorie moderne, le lyrisme a réjoui d'un statut ontologique spécial, au-delà du bien et du mal, pour ainsi dire. Comme la fiction et le discours littéraire en général, l'énonciation poétique serait non-sérieuse, non-engageante.) Les paramètres complexes du genre sont préservés dans la nouvelle poétique mais la posture du locuteur est redéfinie. La démarche transitive de la poésie analysée confirme les prémisses de la « philosophie » dialogique : le dialogue est plus naturel et plus primaire que le monologue, tout soliloque contient des traces dialogiques et se structure sur un axe communicatif, et même la plus solipsiste des énonciations subjectives poétiques n'est souvent que la réélaboration apparemment « autistique »d'un dialogue intérieur.

\section{Carmen Popescu}

cpopescu71@gmail.com

Bulevardul 1 Mai, no. 75, Bl. 25, sc. 2, ap. 2

Craiova

Dolj 200334

ROMANIA

\section{Bibliographie}

Bakhtine, M. 1984. Esthétique de la création verbale. Paris: Gallimard.

Barthes, R. 1984. Le bruissement de la langue. Paris : Seuil.

Benveniste, E. 1966. Problèmes de linguistique générale. Paris : Gallimard.

Bernic, C. 2012. După 40 de ani. Aktionsgruppe Banat. - Observator cultural, 620, avril, http://www. observatorcultural.ro/Dupa-40-de-ani.-AktionsgruppeBanat*articleID_26824-articles_details.html (20.09.2015).

Bessière, J., 2008. La littérature est-elle critique ? - Tracés. Revue de Sciences humaines, Hors-série, Présent et futurs de la critique, 71-99.

Bodiu, A., Bucur, R., Moarcăş, G. 1999. Romanian Poets of the 80's and'90's. A Concise Anthology. Piteşti: Paralela 45.

Crăciun, Gh. 2002. Aisbergul poeziei moderne. Pitești: Paralela 45.

Crăciun, Gh. (ed.). 1999. Competiția continuă. Generația 80 in texte teoretice. Piteşti: Paralela 45.

Crudu, D. 1994. Falsul Dimitrie. Târgu-Mureş: Arhipelag. 
Subjectivité poétique, dialogisme et transitivité

Flora, I. 2004. Trădarea metaforei / La métaphore trahie, traduit par Paul Miclău. Pitești : Paralela 45.

Fromm, W. 1979. Vom Gebrauchswert zur Besinnlichkeit. - Die Woche, 26 janvier.

Flottum, K. 2002. Fragments guillemetés dans une perspective polyphonique. - Tribune 13. Skriftserie for Romansk institutt, Université de Bergen, Eds. K. Fløttum \& H. V. Holm, http://www.hum.au.dk/romansk/polyfoni/Diverse\%20 artikler/Flottum_guillemets.htm (20.09.2015).

Gogea, V. 1999. "Falsul Dimitrie" sau adevăratul Dumitru Crudu. - Contrafort, 1112, 61-62 ; https://vasilegogea.wordpress.com/2010/06/19/falsul-dimitrie-sauadevaratul-dumitru-crudu/ (20.09.2015).

Ilea, L. 1999. une belle journée de printemps. en plein champ. - A. Bodiu, R. Bucur, G. Moarcăş, Romanian Poets of the 80's and'90's. A Concise Anthology. Piteşti: Paralela 45, 307.

Jenny, L.2003. Méthodes et problèmes. La Poésie. Dpt. de Français moderne - Université de Genève, http://www.unige.ch/lettres/framo/enseignements/methodes/ elyrique/elintegr.html (20.09.2015).

Kerbrat-Orecchioni, C. 2002 [1980]. L'énonciation: de la subjectivité dans le langage. Paris: Armand Colin.

Kristeva, J. 1969. Séméiotiqué. Recherches pour une sémanalyse. Paris : Seuil.

Lefter, I. B. 1995. La reconstruction du moi de l'auteur. - Euresis. Cahiers roumains d'études littéraires. Le postmodernisme dans la littérature roumaine, 1-2. Bucarest : Univers, 168-171.

Maio, S. 2005. Creating Another Self: Voice in Modern American Personal Poetry. Missouri: Truman State University Press.

Marin, M. 1991. Atelierele (1980-1984). București: Cartea Românească.

Mușina, A. 1995. Le postmodernisme aux portes de l'Orient. - Euresis. Cahier roumains d'études littéraires. Le postmodernisme dans la littérature roumaine, 1-2. Bucarest : Univers, 155-167.

Parpală, E. 2011. Ioan Flora - un postmodern atipic. - E. Parpală, C. Popescu, eds., Postmodernismul. Creație și interpretare. Craiova: Universitaria, 73-90.

Popescu, C. 2013. Metacommunication as Ritual: Romanian Contemporary Poetry. - R. D. Sell, A. Borch, I. Lindgren, eds., The Ethics of Literary Communication: Genuineness, Directness, Indirectness. Amsterdam/ Philadelphia: John Benjamins, 147-166.

Popescu, C. 2014. Subjectivity and the Dialogic Self: The Christian Orthodox Poetry of Scott Cairns and Cristian Popescu. - R. D. Sell, ed., Literature as Dialogue: Invitations Offered and Negotiated. Amsterdam/ Philadelphia: John Benjamins, $197-218$.

Sarfati, G.-E. 2009. Eléments d'analyse du discours. Paris : Armand Colin.

Stăniloaie, D. 1987. Chipul nemuritor al lui Dumnezeu. Craiova : Editura Mitropoliei Olteniei.

Vianu, T. 1988. Arta prozatorilor români. București : Minerva. 